\title{
Device and Method for Measuring Volume in Receptacles for Storing Wine
}

\author{
L Castañeda* \\ Postgraduate Studies and Research Section of the Higher School of Medicine, Mexico
}

*Corresponding author: Luis Castañeda, Sección de Estudios de Posgrado e Investigación de la Escuela Superior de Medicina, Instituto Politécnico Nacional, Plan de San Luis yDíaz Mirón S/N, Casco de Santo Tomás, Cd. de México, México, C. P. 11340.

\section{ARTICLE INFO}

Received: 聎 June 07, 2021

Published: 慧 June 14, 2021

Citation: L Castañeda. Device and Method for Measuring Volume in Receptacles for Storing Wine. Biomed J Sci \& Tech Res 36(3)-2021. BJSTR. MS.ID.005858.

Keywords: Wine Boxes; Wine Containers; Wine Volume Measurement; Volume Measurement Technology

\section{ABSTRACT}

This study is on the design and method of a volume measuring device installed inside a box used to store liquids mainly wine. Current devices that store wine in boxes have inside these boxes, inner boxes regularly made of rigid cardboard and a sealed plastic receptacle, which has an outlet tap to serve the wine. The device is focused on being used in the new wine packaging in a cardboard box. The measuring device is placed at the bottom of the wine dispenser box and displays a measurement of the volume of the wine even in the box via a measurement tower. The wine storage box contains inside: the device located at the bottom of the box containing the wine (i.e., the liquid), and the plastic container containing the wine above our measuring device. The measuring device will feel the weight of the current wine content in the plastic container and will automatically move a signal indicating the level of the liquid even in the plastic container. The measurement will be visible from the outside of the box by the consumer, the device is included by a receptacle or sealed bag connected to the measurement tower. The weight measurement receptacle is sealed and made of a material with a leak-resistant layer, and which is usually irregularly oval and is filled with at least one form of gas or air and where the packing layer is shaped to fill the bottom of the container and it is placed under the bag for storage and shipping. As the pressure of the liquid weight changes, the receptacle reacts by moving a measuring marker that reflects such changes, thus measuring the current volume of the liquid in the shipping box. There are numerous patents for measuring liquids, particularly for wine, and then we present several of these patents that are the most relevant to our case.

\section{Introduction}

The Beavis patent [1] is a flow sensor that includes a fluid chamber configured to receive the fluid. A diaphragm assembly is configured to be moved when the fluid inside the fluid chamber is displaced. A translator assembly is configured to monitor the displacement of the diaphragm assembly and generate a base signal, at least in part, once the amount of liquid is displaced within the fluid chamber. It is therefore a device for measuring fluid in a rigid tank. Measuring the volume of liquid in a wine or liqueur bottle is Palmquist's patent [2]. It consists of a sensor system and liquid level report for a bar, having a liquid sensor in wireless communication with a remote computer that has the software and algorithms to report the volume. The sensor is substantially the shape of a wine bottle stopper, which includes the means to measure the height of the liquid in the bottle, bottleneck orientation detection, and wirelessly sending data to the receiver. The receiver is associated with a computer that calculates the volume of the liquor based on changes in the height of the liquid in the bottle from pre-served to post-served status. The software extrapolates this information to produce a variety of real-time reports on consumption, including reports of errors caused by over-serving, serving less and possible theft.

The invention of Augustine M et al. [3] provides methods and devices for the analysis of sealed containers of consumables using Magnetic Nuclear Resonance Spectroscopy (MRI). The high static 
radio frequency used by magnetic fields is used in MRI experiments that in no way affects the quality of the food or drink examined by the methods used here [4]. An interesting but technically and economically impractical solution for measuring volume in containers used by the consumer in general. Augustine's invention [5] provides non-invasive and non-destructive analytical methods for determining the level of wine acidification. These methods and devices of this invention can be routinely used in the evaluation of wine quality and studies of wine cork aging. Moreover, these intact bottle analysis methods are not limited to determining the deterioration and content of acetic acid in wines but may be extended to the study of other molecular components of the wine (e.g., aldehydes and flavonoids), as well as components and/or contaminants in other types of sealed consumables. A good patent for the analysis of wine in a container. This patent does not handle volume issues.

As can be seen in all the patents discussed above are for the measurement of liquids, for wine. However, most if not all are about volume measurements for bottles or tanks. Unfortunately, none of them are focused on the new wine packaging in a cardboard box. The wine is not in contact with the cardboard box but a sealed plastic container and with a single outlet of the wine-pumping outlet tap As the boxes are closed, there is no way for the consumer to determine the amount of leftover wine in the container, just an approximation based on the weight of the box. If you are good at estimating or remembering the initial weight of the box and the current weight, then you will have no problems determining the amount of wine remaining in the box. However, most people are not trained or are not good estimators of the initial weight of the box and compare it to the new weight.

Several patents have devices like those proposed here but used differently. Here are some examples of these patents. Curry David P's patent [6] in general these are level indicators, more particularly indicators for fluid level in a storage tank or container. It is related to our idea, but it is only for containers or tanks with rigid structures, where we have product containers in non-rigid plastic bags. Curry [7] in its patent provides an intravenous (IV) airbag containing a specialized device, referred to as the airless intravascular infusion device (AIVID). AIVID allows one to see the amount of fluid in the IV bag, but substantially decreases or completely prohibits the risk of inadvertent infusion of air in a patient. Example of the use of plastic bags for product handling and visual measurement.

One more patent on fluid measurement in a rigid-walled container presents Fang Yun, et al. [8]. A vessel comprising a surface that confines the fluid, a basal surface arranged below the surface that confines the fluid, and a light guide. The light guide includes a first window that partially defines the basal surface and a second window that partially defines the surface that confines the fluid, where the second window is arranged over a lower region of the surface that confines the fluid. A patent containing an external visual meter in a container with rigid walls presents Tai Yuen Wa [9]. In a liquid storage container, it has a lower-body to store the liquid, an outer surface, and a higher part defining a central opening at the dispenser. A handle is attached to the body at a first default location. A liquid level indicator is attached to the body at a second default location that is at a default distance from the first default location. The liquid level indicator has a passage of fluid through it that is in fluid communication with the inside of the body. The liquid level indicator has a first end attached to the body, a second end attached to the body, and a substantially transparent part between the first and second ends.

Sisk Henry B, et al. [10] patented a device to determine the deviation of a volume of liquid from a pre-determined volume comprises the main container to receive the liquid and a measuring vessel from which the deviation of the liquid meniscus in the measuring vessel is determined. A communication tube that extends from the neck of the main vessel communicates the main vessel with the secondary vessel through a ball valve. Liquid over a landfill in the neck flows into the measuring vessel. The liquid volume deviation is read on a scale adjacent to the measuring vessel. A variable-volume bladder filled with liquid alcohol is mounted in the main container to compensate for volume changes in the main vessel due to temperature variations. This patent is also on rigidwalled containers.

The art closest to what is proposed by us is Karlsson's patent [11] which is an innovation that refers to a level indicator to read the level of a liquid, preferably wine, in a bag-in-box. The indicator comprises a bag containing a liquid and a tube connected to the bag. The level indicator bag is placed between the box and the bag containing the wine and the tube can be placed vertically. The liquid rises in the tube in proportion to the weight of the wine in the bag and the level in the tube reveals the level of the wine in the bag. One of the basic differences of our proposal with this patent is in the use of air or gas to handle the level indicator since Karlsson's patent uses liquid for this process. The study proposed here solves the problem of estimating how much was still in the box, just by looking at the measuring device proposed here. It is important to mention that the article presented here is the basic compilation of the patent application against the Mexican Institute of Industrial Property [12], which is a legal continuation of the patent granted in the United States [13].

\section{Device and Method}

The work presented here is on a volume measuring device installed inside a box that is used to store liquid. Figure 1 shows a barrel (\#1) that has a volume measuring device inside it. The volume measuring device consists of several connected elements (See Figure 2): a measuring tower (\#4), a constrictor channel (\#5), 
and the receptacle (\#6). The measuring tower \#4 is inserted into the wall 3 (see Figure 1 number 1 ) so that the tower is visible outside the box. Figure 3 shows the \#2 volume measuring device, consisting of a \#4 measuring tower, a \#5 constrictor channel, a \#6 receptacle, a volume \#7 indicator, and various volume measurement signals \#8 by Half Full, \#9 by Vacuum and \#10 per Full. The complete appliance 2 is filled with air and sealed. The amount of air will depend on the maximum weight it supports.
It will be done in such a way that when the maximum weight is above receptacle 6 , the volume indicator will be at the highest level indicating a full load \#10. When the receptacle is half full, volume indicator \#7 will be in the middle mark \#8, and when the receptacle is empty, volume indicator \#7 will be in the Vacuum mark \#9. The constrictor \#5 channel will cause the \#7 volume indicator to drop into the impossible \#6 receptacle since volume indicator \#7 does not fit through the constrictor channel \#5.

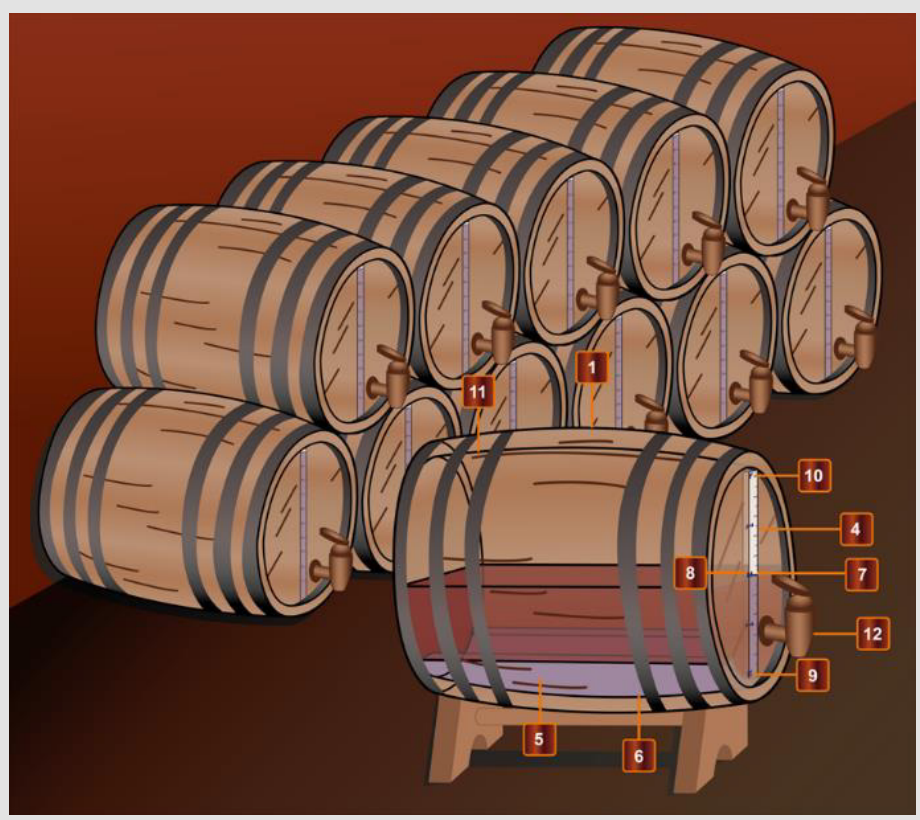

Figure1: Volume Measurement Device Inside your Container.

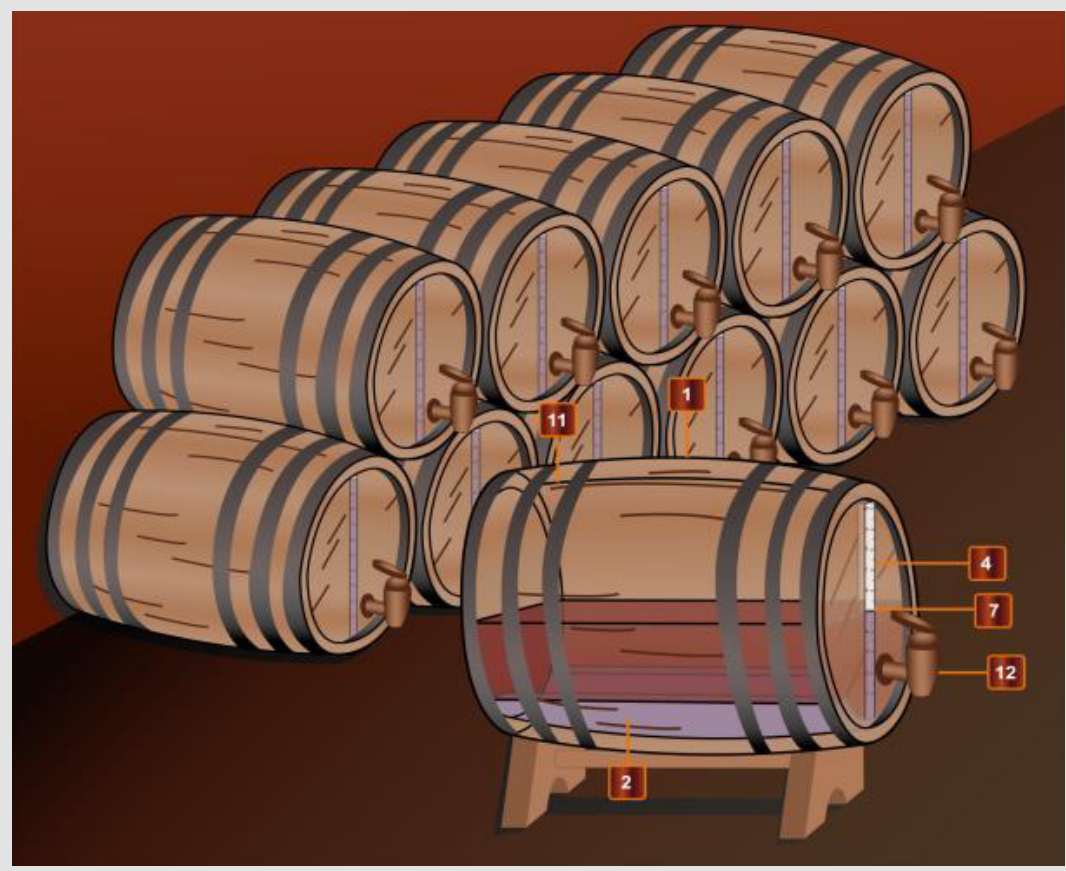

Figure 2: Volume measuring device with liquid inside. 


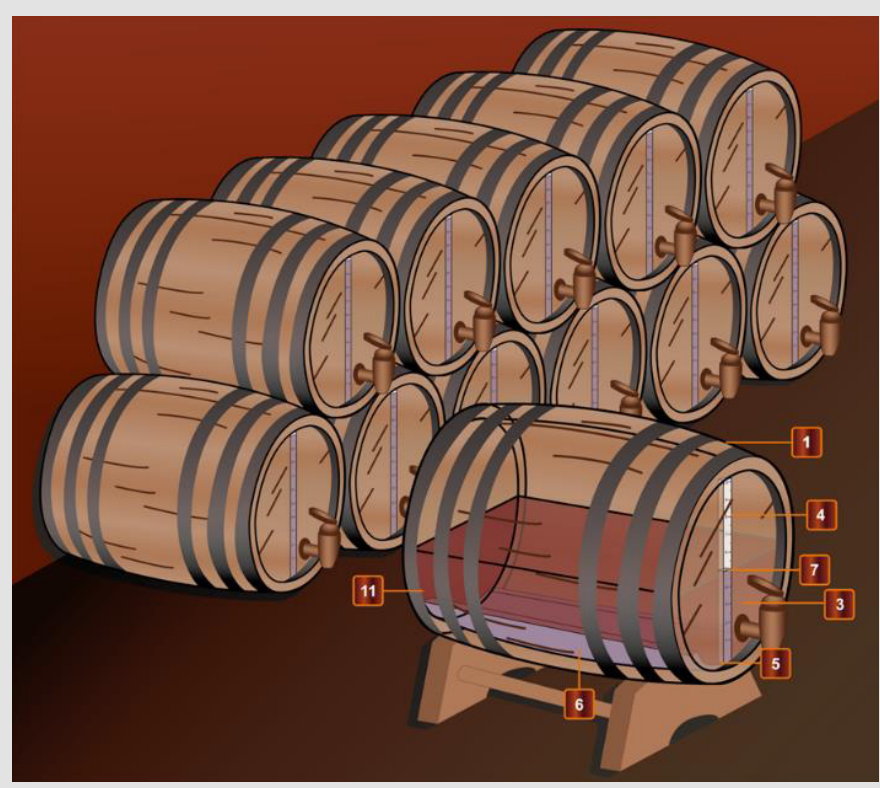

Figure 3: Volume Measurement Device with all its parts.

When the volume measuring device is properly placed in the box as shown in Figure 1, then when the box is filled with the liquid to be dispensed in a sealed plastic bag and with a ball valve, the weight of the liquid will cause the air inside the receptacle to move through the constrictor channel towards the measuring tower, causing the volume indicator to move upwards until it reaches the Full mark. When the liquid is served, the weight will decrease, lower the pressure on the receptacle, which will cause the gravity volume marker to move down respectively in the measuring tower. The device declaration and method described here represent the preferential form of the invention; however, variations of this in the form, construction, and arrangement of its components and the application of the modified invention are possible without going out of the spirit and scope of the attached claims.

\section{Claims}

With the idea of making this article as complete as possible, we present here the claims that were used in the application of patent before the Mexican Institute of Industrial Property [12]. Having described the invention sufficiently, the contents of the following clauses are the claims of this work:

a) A device for measuring and serving liquids including: a container, a bag for storing and shipping liquids and a device for measuring volume; where the aforementioned bag for storing and shipping liquids is located inside the container; where the walls of the container are made of either one rigid or substantially rigid; where the above-mentioned volume measuring device is composed of a measurement tower, a constrictor channel and a weight measurement receptacle; where the aforementioned weight measurement receptacle is sealed and made of a material with a leak-resistant layer and which is usually irregularly oval in shape and is filled with at least one form of gas or air and where the packing layer is shaped to fill the bottom of the container and is placed under the bag for storage and shipping; where the measurement tower is in fluid communication with the constrictor channel and the weight measurement receptacle; where the measurement tower has a graduated scale positioned along the measurement tower and how to indicate the volume of the interior of the measurement tower; where the aforementioned movement of the aforementioned way of indicating weight, volume or relative fill level is synchronized with the pressure of the displacement of air or gas exerted on the aforementioned weight measurement receptacle by putting the weight of the aforementioned bag to store and dispatch liquids in the weight measurement receptacle; where the way to indicate weight, volume or relative fill level of the displaced air or gas will move between and starting at the final narrow by connecting the constricting channel and moving towards the end of the measuring tower opposite the constricting connection and proportional to the weight, volume or relative fill level of the liquid in the bag to store and dispatch liquids; where the aforementioned measuring tower will have a substantial form of $\mathrm{L}$ bent in the aforementioned final narrowness of the aforementioned constrictor channel; where the aforementioned tower is recessed on one of the external sides of the aforementioned container presenting a substantially flat surface that provides a visual view of the means to indicate weight, volume or relative level of fill of the liquid in the bag to store and issue liquids along the gradual scale and that is visible externally to the container. 
b) The device of claim 1 where the final narrow that connects the measurement receptacle with the measurement tower can be extended such that it can be curved around obstacles in the container where the measurement receptacle will be placed to measure weight, volume, or relative fill level within the bag to store and dispatch liquids.

c) The device of claim 1 where the graduation of measurements contains at least five measurements indicating proportions of liquid in the container.

d) The device of claim 3 where the measurement graduation scale is read in measurements of Vacuum, Full, Medium, 1/4, and $3 / 4$.

e) The device of claim 1 where the container is shaped like a box, cylinder, or parallelepiped.

f) The device of claim 5 where the opposite side of the graduated measurement scale of the measurement tower contains increments marked with full and half values starting from zero to the total number of bottles that the bag for storing and issuing liquids can store.

g) The device of claim 1 where the bag for storing and issuing liquids contains beverages, wines, or alcoholic beverages such as liquid to be stored and dispatched.

h) The device of claim 1 where the volume meter is built as a single unit.

i) The device of claim 1 where the bag for storing and shipping liquids contains a faucet to serve liquid.

j) A method for measuring the volume of the contents and serving liquid using a volume measuring device including: a container a bag for storing and shipping liquids and a device for measuring volume; where the aforementioned bag for storing and shipping liquids is located inside the container; where the walls of the aforementioned container are made of either one rigid or substantially rigid; where the above-mentioned volume measuring device is composed of a measurement tower, a constrictor channel and a receptacle of weight measurements; where the aforementioned weight measurement receptacle is sealed and made of a material with a leak-resistant layer and which is usually irregularly oval in shape and is filled with at least one form of gas or air and where the packing layer is shaped to fill the bottom of the container and is placed under the bag for storage and shipping; where the measurement tower is in fluid communication with the constrictor channel and the weight measurement receptacle; where the aforementioned measurement tower has a graduated scale positioned along the measurement tower and how to indicate the volume of the interior of the measurement tower; where the movement of the way of indicating weight, volume or relative fill level is synchronized with the pressure of the displacement of air or gas exerted on the aforementioned weight measurement receptacle by putting the weight of the bag to store and dispatch liquids in the weight measurement receptacle; where the way to indicate weight, volume or relative fill level of the displaced air or gas will move between and starting at the final narrow by connecting the constricting channel and moving towards the end of the measuring tower opposite the constricting connection and proportional to the weight, volume or relative fill level of the liquid in the bag to store and dispatch liquids; where the measuring tower will have a substantial form of $\mathrm{L}$ bent in the final narrowness of the constrictor channel; where the tower is recessed on one of the external sides of the container presenting a substantially flat surface that provides a visual view of the means to indicate weight, volume or relative fill level of the liquid in the bag to store and issue liquids along the gradual scale and that is visible externally to the container.

k) A system for measuring the volume of the content and serving liquid using a volume measuring device including: a container a bag for storing and shipping liquids and a device for measuring volume; where the aforementioned bag for storing and shipping liquids is located inside the container; where the walls of the container are made of either one rigid or substantially rigid; where the above-mentioned volume measuring device is composed of a measurement tower, a constrictor channel and a weight measurement receptacle; where the weight measurement receptacle is sealed and made of a material with a leak-resistant layer and which is usually irregularly oval in shape and is filled with at least one form of gas or air and where the packing layer is shaped to fill the bottom of the container and is placed under the bag for storage and shipping; where the measurement tower is in fluid communication with the constrictor channel and the weight measurement receptacle; where the measurement tower has a graduated scale positioned along the measurement tower and how to indicate the volume of the interior of the measurement tower; where the movement of the aforementioned way of indicating weight, volume or relative fill level is synchronized with the pressure of the displacement of air or gas exerted on the weight measurement receptacle by putting the weight of the bag to store and dispatch liquids in the weight measurement receptacle; where the way to indicate weight, volume or relative fill level of the displaced air or gas will move between and starting at the final narrow by connecting the constricting channel and moving towards the end of the measuring tower opposite the constricting connection and proportional to the weight, volume or relative fill level of the liquid in the bag to store and dispatch liquids; where the aforementioned measuring tower will have a substantial form of $\mathrm{L}$ bent in 
the final narrowness of the constrictor channel; where the tower is recessed on one of the external sides of the container presenting a substantially flat surface that provides a visual view of the means to indicate weight, volume or relative level of fill of the liquid in the bag to store and dispense liquids along the gradual scale and that is visible externally to the container.

\section{Results from Discussion}

This work presents an innovation to an existing need in an emerging market such as the packaging of wines in cardboard boxes. A thorough study of the previous art related to the patent proposal was conducted, all possible interferences to existing patents were analyzed and a device and method were designed that was unique and not existing in the market. This work is the result of this study and the development of the patent in Mexico and the United States.

\section{Conclusions}

With this device and method, we will be presenting an innovation to wine producers and allowing them to be the first on the market using a useful and simple device.

\section{Acknowledgments}

The authors gratefully acknowledge the financial support from the Escuela Superior de Medicina del Instituto Politécnico Nacional, through Project no. 20210385.

\section{ISSN: 2574-1241}

DOI: 10.26717/BJSTR.2021.36.005858

L Castañeda. Biomed J Sci \& Tech Res

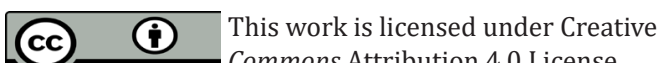

Submission Link: https://biomedres.us/submit-manuscript.php

\section{References}

1. Beavis R (2016) Product dispensing system, US Patent 9,488,510.

2. Palmquist K (2013) Liquid level measuring device, US Patent $8,453,878$.

3. Augustine M, Matthew P, Bruins, Paul, Weekly, et al. (2006) Methods and devices for analysis of sealed containers, US Patent 7,012,427. Insight.

4. Pykett I, TW Skloss, MJ Hennessy (2001) Method for non-invasively and without contact, inspecting foil enclosed packages, using magnetic resonance techniques, US Patent 6,333,629.

5. Augustine M (2005) Methods and devices for analysis of sealed containers, US Patent 6,911,822.

6. Curry David P, Stetson Douglas, Dockendorff James B (2018) Visual liquid level indicator, United States Patent \#10,156,467.

7. Curry Jeremy Scott (2012) Airless intravenous bag, United States Patent $\# 8,251,952$.

8. Fang Yun, Morelock David Michael, Masalkar Prafulla (2012) Sensing the amount of liquid in a vessel, United States Patent \#8,136,690.

9. Tai Yuen Wa (2009) Thermal liquid container having liquid level indicator, United States Patent \#7,603,901.

10. Sisk Henry B, Madden John J, O’Sullivan, Niall A, Comerford, et al. (1997) Liquid volume measuring apparatus, United States Patent \#5,606,109.

11. Karlsson Olof (2008) Device for Indicating the Level of a Liquid in a Bag-In-Box, World Intellectual Property Organization (WIPO) WO/2008/100199.

12. Gonzalez Carlos (2019) Mediciones Continuas De Volumen En Receptáculos Para Líquidos, México Solicitud MX/a/2019/010507.

13. Gonzalez Carlos (2019) Continuous Volume Measurement on a Receptacle for Liquids, US Patent \#10,352,749.

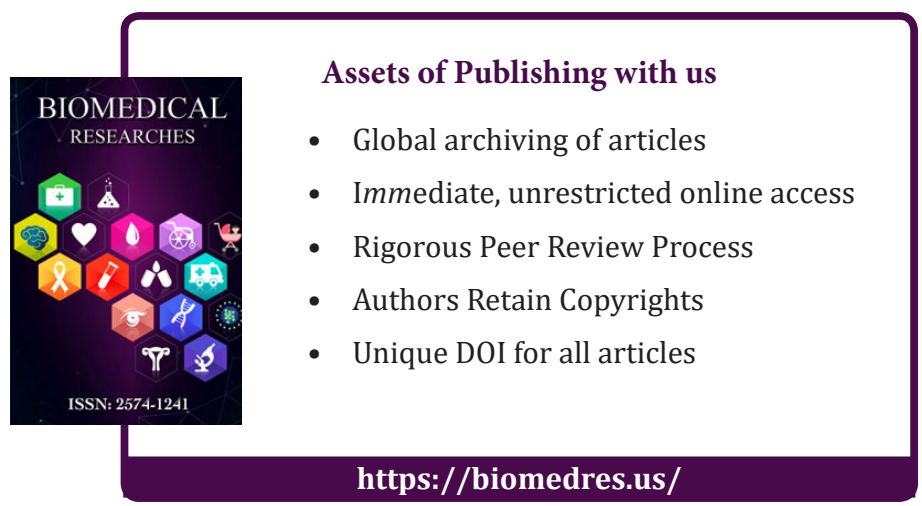

